\title{
Birth Weight In Relation To Obesity And Diabetes Gene Expression In Healthy Infants; A Case- Control Study
}

\section{Sahar Cheshmeh}

Kermanshah University of Medical Sciences

Shima Moradi ( $\nabla$ Shima.Moradi@kums.ac.ir)

Kermanshah University of Medical Sciences

Seyyed Mostafa Nachvak

Kermanshah University of Medical Sciences

\section{Arman Mohammadi}

Shahid Beheshti University of Medical Sciences

\section{Azadeh Erfanifar}

Jundishapur University of Medical Science

Arezoo Bajelani

Azad Islamic University

\section{Research Article}

Keywords: low birth weight, obesity, diabetes, gene expression

Posted Date: June 2nd, 2021

DOI: https://doi.org/10.21203/rs.3.rs-537950/v1

License: (1) This work is licensed under a Creative Commons Attribution 4.0 International License. Read Full License 


\section{Abstract}

Background: Since obesity and diabetes are a prevalent phenomenon in the world, recognizing the factors affecting these two conditions can be effective in modifying them. We decided to investigate the expression of obesity and diabetes genes in infants with birth weight lower than $2500 \mathrm{~g}$.

Methods: The current case- control study was performed on 215 healthy infants aged 5-6 months which referred to health and treatment centers of Kermanshah. After measuring weight and height of the infant, the values were compared with the diagram of WHO to assure being healthy and well-grown and infants who were healthy, were selected to the study. 78 infants was in case group and 137 infants was in control group. $5 \mathrm{cc}$ of intravenous blood was collected from all infants. Blood samples were collected in Ethylenediaminetetraacetic acid (EDTA) coated vials to evaluate the expression of MC4R, MTNR1B, PTEN, ACACB, PPAR-Y, PPAR-a, (NRXN3 (NTRK2), (PCSK1), (A2BP1), (TMEM18), (LXR), (BDNF), (TCF7L2), $(F T O)$ and $(C P T 1 A)$ genes. The data was analysed by Chi square, Mann-Whitney $U$ and Spearman tests.

Results: An inverse significant correlation between birth weight and obesity and diabetes genes including MTNR1B, NTRK2, PCSK1, and PTEN genes ( $\mathrm{r}=-0.221,-0.235,-0.246$, and -0.418 , respectively). In addition, the expression level of the mentioned genes in the LBW infant was significantly up-regulated than the normal weight infants $(P=0.001,0.007,0.001$, and $<0.001$, respectively). The expression level of $P P A R-a$ gene had significantly positive correlation with the birth weight $(r=0.19, \mathrm{P}=0.005)$. The expression level of PPAR-a gene in the normal weight infants was significantly up-regulated than the LBW infants $(\mathrm{P}=$ $0.049)$.

Conclusion: Expression level of MTNR1B, NTRK2, PCSK1, and PTEN genes up-regulated in the LBW infants, however, the expression level of PPAR-a gene was significantly down regulated in the LBW infants compared to the infants with normal birth weight.

\section{Introduction}

Low birth weight (LBW) is enhanced short-term and long-term consequences such as infant mortality, cognitive disorders, growth failure, and is associated with obesity and diabetes in adulthood [1,2]. According to definition by World Health Organization, LBW infants refers to infants whom their birth weight is less than 2500 grams [3]. Approximately $15-20 \%$ of births are related to LBW infants which annually accounts for about 20 million births [4].

Some studies have found a U-shaped or J-shaped association between birth weight and obesity in adulthood, although these findings have been contradictory $[5,6]$. On the other hand, it has been suggested that LBW can increase the risk of developing diabetes in adulthood [5]. Since obesity and diabetes are a prevalent phenomenon in the world, recognizing the factors affecting these two conditions can be effective in modifying them $[7,8]$. 
Both environmental and genetic factors are involved in the pathogenesis of obesity and diabetes [8-10]. Among the genes involved in the etiology of obesity and diabetes can be named Melanocortin 4 receptor $(M C 4 R)$, Melatonin Receptor 1B (MTNR1B), Peroxisome proliferator-activated receptor gamma (PPAR- $\gamma)$, Phosphatase and tensin homolog $(P T E N)$, Acetyl-CoA Carboxylase Beta $(A C A C B)$, and peroxisome proliferator-activated receptor-a (PPAR-a), $M C 4 R$ gene encodes melanocortin receptor, $\mathrm{MC}_{4}$ protein, a Gprotein receptor that high it's expression is related to body fat distribution and energy intake in children. MTNR1B gene is located on chromosome 11q21 and synthesis melatonin receptor 2 in which this gene is related to all diabetes types including diabetes mellitus, type 1 diabetes, and gestational diabetes $[11,12]$. In addition, down- regulated of PTEN and $A C A C B$ genes can decrease blood sugar and subsequently prevent diabetes and obesity $P T E N$ is a phosphatase which plays role in signaling pathway and suppression of tumor $[10,11,13]$. $A C A C B$ is a biotin dependent enzyme which catalyzes irreversible carboxylation of acetyl COA to manolyl CoA and is effective in obesity and diabetes by reducing fatty acids oxidation and increasing of insulin resistance $[14,15]$. Peroxisome proliferative activating receptors (PPARs) are part of the nuclear hormone receptors [16].

Up-regulated of expression level of PPAR- $y$ regulates the secretion of adipose tissue hormones and reduces insulin resistance [17]. In addition, PPAR- $a$, which is expressed mostly in tissues with a high level of fatty acid catabolism, therefore up-regulated of PPAR- $a$ is involved in reducing obesity and body fat [18]. Since the epidemiologic studies showed that birth weight can play role in incidence of the obesity and diabetes, this study aimed to investigate the expression of obesity and diabetes genes in infants with birth weight lower than $2500 \mathrm{~g}$.

\section{Methods And Materials}

The current case control study was performed on healthy infants aged 5-6 months which referred to health and treatment centers of Kermanshah. Sample size was calculated based on the weight mean of children in study by Zarrati et al. [19] using the sample size formula for case studies with $90 \%$ power and $95 \%$ confidence in each group of 45 infants. Inclusion criteria were healthy infants, lack of metabolic diseases, not using medications in last month, breastfeeding or using infant milk (formula), not initiating supplementary nutrition, healthy parent and lack of metabolic disease of their parents. Based on birth weight, we considered infants with birth weight less than $2500 \mathrm{~g}$ in the case group and weight between 2500-4000 $\mathrm{g}$ in the control group [3]. For more reassurance, we entered 80 infants in the case group and twice as many in the control group, which due to the fact that the information of some samples was incomplete, finally 78 infants remained in the control group and 138 infants in the control group.

\section{Ethical consideration}

At first, the process of the study was completely explained for the parents, and informed written consent was obtained from their parents. This study was approved by the ethics committee of Kermanshah University of medical science (ethical number: IR.KUMS.RES.1397.081). 
Anthropometry

Firstly the health information of infant including term or preterm, birth height, weight, and head circumference, and the food kind was recorded in the questionnaire. After that, the infants' height was measured by tape and in supine position and the infants weight was measured with the least cloth and without diaper. After measuring weight and height of the infant, the values were compared with the diagram of WHO to assure being healthy and well-grown and infants who were healthy, were selected to the study.

Sampling and expression of obesity and diabetes genes

$5 \mathrm{cc}$ of intravenous blood was collected from all infants. The blood sample was then centrifuged at 500 rpm for 15 minutes. The serum isolated from the blood sample was frozen at minus $-80^{\circ} \mathrm{C}$. Blood samples were collected in Ethylenediaminetetraacetic acid (EDTA) coated vials to evaluate the expression of MC4R, MTNR1B, PTEN, ACACB, PPAR- $y, P P A R-a$, Neurexin-3-alpha (NRXN3), Neurotrophic Receptor Tyrosine Kinase 2 (NTRK2), Proprotein convertase 1(PCSK1), Ataxin-2 binding protein 1 (A2BP1), Transmembrane 18 gene (TMEM18), Liver $X$ receptor $(L X R)$, Brain-derived neurotrophic factor $(B D N F)$, transcription factor 7-like 2 (TCF7L2), fat mass and obesity-associated (FTO), and carnitine palmitoyltransferase IA (CPT1A) genes. Using Ficoll-Histopaque solution gradient, peripheral blood mononuclear cells (PBMC) were isolated during density gradient centrifugation (Ficoll-paque, Miltenyi Biotec $\mathrm{GmbH}$, and Germany). Using Trisor Regaent kit, total RNA from PBMC cells were extracted (Iranian pure YTzol RNA). One microgram of extracted RNA was applied for complementary DNA synthesis (cDNA) by Prime Script-RT Reagent kits according to the manufacturer's instructions (Takara Bio Ink. Tokyo, Japan). Dedicated primers purchased and designated from Metabion (Metabion, Germany). (Table 1) Data were normalized as housekeeping genes by 2-tCt expressing 18s-rRnan. All samples were done in three versions.

Table 1. Primers sequences for RT-PCR amplification 
Gene name and symbol $(5 \rrbracket \rightarrow 3 \square)$

MC4R

F: 5囚-CTG ATG GAG GGT GCT ACG AG-3!

R :5邓-TGG GTG AAT GCA GAT TCT TGT T-3!

MTNR1B F : 5®-GCA TGG CCT ACC ACC GAA TC-3!

R : 5囚-AAT AGA TGC GTG GGT CGT ACT-3!

TMEM18 F : 5囚-TGT TAA AGT CGA TGG TGT AGC TC-3[

R : 5囚-GTC CTT GTC CGG TTG TGA ACT-3ם

TCF7L2 F:5囚-

CGGCGAGTCTATGCCACTAT-3ם

$\mathrm{R}: 5 \rrbracket-$

ACACAGGGACCGAGTAATGC-3[

NRXN3 F : 5ه-AGG GGA AAA TTG GAG TTG TCT TC-3[

R : 5囚-CCG TCA TTT ACA GGG GTT CTC T-3

NTRK2

F: 5囚-ACC CGA AAC AAA CTG ACG AGT -3ם

R: 5囚-AGC ATG TAA ATG GAT TGC CCA-3!

PCSK1 F: 5囚-ACC CGA AAC AAA CTG ACG AGT -3!

R: 5囚-AGC ATG TAA ATG GAT TGC CCA-3ロ

A2BP1 F: 5囚-ATTCAAACTACTGCCACC3\-3》

R: 5囚-TGTCTAACACCATCTGCTT$3 \rrbracket$

$18 s$ rRNA
$F: 5 \rrbracket-$

ACCCGTTGAACCCCATTCGTG A-
Gene name and symbol Sequence $(5 \square \rightarrow 3 \square)$

BDNF F:5-GGCTTGACATCATTGGCTGAC-3]

R :5-TGTGCAGTGTGAGAAAGGCTT-3[

ACACB F : 5-CAAGCCGATCACCAAGAGTAAA-3

R : 5-CCCTGAGTTATCAGAGGCTGG-3[

PTEN F : 5-

CAAGATGATGTTTGAAACTATTCCAATG$3 \rrbracket$

R : 5-CCTTTAGCTGGCAGACCACAA-3[

LXR-a F :5-CCTTCAGAACCCACAGAGATCC-3[

R :5-ACGCTGCATAGCTCGTTCC-3ロ

PPAR-y F :5-GATGCCAGCGACTTTGACTC-3[

$\mathrm{R}: 5-A C C C A C G T C A T C T T C A G G G A-3 \square$

FTO

F :5-ACTTGGCTCCCTTATCTGACC-3[

R :5-TGTGCAGTGTGAGAAAGGCTT-3[

CPT1A F :5-TCCAGTTGGCTTATCGTGGTG-3[

R :5-TCCAGAGTCCGATTGATTTTTGC-3ロ

PPAR-a F :5-ATGGTGGACACGGAAAGCC-3[

$\mathrm{R}: 5$-CGATGGATTGCGAAATCTCTTGG$3 \rrbracket$
$\mathrm{F}$, forward 
$\mathrm{R}: 5 \rrbracket-$ GCCTCACTAAACCATCCAATCGG$3 \rrbracket$
$\mathrm{R}$, reverse

\section{Statistical analysis}

In current study all variables were analyzed by SPSS (SPSS Inc. Chicago, IL, USA version 19) and STATA (Stata Corp, College Station, TX, version 14). The data normality was checked using Kolmogorov-Smirnov test. Basic characteristics of infants described by mean, frequency, and percent frequency. Chi square test was used to compare qualitative variables. Mann-Whitney $U$ and Spearman correlation tests were used to evaluate the expression of obesity and diabetes genes in peripheral blood cells and its relationship with birth weight. In all tests, a significance level of less than 0.05 was considered.

\section{Results}

In this present study, 137 infant and 78 infant were enrolled in the control and case studied groups, respectively. In term of gender of the infants, food kind of the infants, their mother age, their mother weight before pregnancy, their mother and father BMI were not seen any difference between two studied groups. The mean of weight in control and case groups were $3.18 \pm 0.27$ and $1.79 \pm 0.48 \mathrm{~kg}$, respectively $(P<0.001)$. All characteristics of infants are presented in Table 2.

Table 2. Basic characteristics of infants based on the birth weight 


\begin{tabular}{|llll|}
\hline Variables & Normal weight & Low birth weight & \multirow{2}{*}{ P1 } \\
\cline { 2 - 3 } & $(\mathrm{n}=137)$ & $(\mathrm{n}=78)$ & \\
\hline Gender, boy \% & 54 & 51.3 & 0.403 \\
\hline Preterm, \% & 0 & 83.3 & $<0.001$ \\
\hline Food kind, \% & & & \\
\hline Breast feeding & 34.3 & 30.8 & 0.09 \\
\hline Formula & 35 & 24.4 & \\
\hline Mixed & 30.7 & 44.9 & $<0.001$ \\
\hline Birth weight, cm & $3.18 \pm 0.27 *$ & $1.79 \pm 0.48$ & $<0.001$ \\
\hline Birth height, cm & $51.87 \pm 3.71$ & $42.92 \pm 5.30$ & $<0.001$ \\
\hline Head circumference, cm & $33.69 \pm 2.49$ & $28.54 \pm 2.85$ & $<0.001$ \\
\hline Current weight, kg & $7.87 \pm 0.83$ & $6.43 \pm 1.4$ & 0.186 \\
\hline Mother age, years & $27.29 \pm 5.86$ & $28.41 \pm 6.32$ & 0.359 \\
\hline Mother weight before pregnancy, kg & $69.7 \pm 10.09$ & $75.26 \pm 13.13$ & $<0.001$ \\
\hline Pregnancy weight gain, kg & $14.18 \pm 4.05$ & $9.47 \pm 8.63$ & 0.746 \\
\hline Mother BMl, kg/m ${ }^{2}$ & $26.62 \pm 4.09$ & $25.74 \pm 4.01$ & 0.739 \\
\hline Father BMl, kg/m ${ }^{2}$ & $26.17 \pm 3.14$ & $26.12 \pm 3.04$ & 0.257 \\
\hline Mother smoking, \% & 2.2 & 0 & \\
\hline
\end{tabular}

*Mean \pm SD

P1 was obtained Chi square and Mann-Whitney U Tests.

In current study, we found that there was an inverse significant correlation between birth weight and obesity and diabetes genes including MTNR1B, NTRK2, PCSK1, and PTEN genes $(r=-0.221,-0.235,-0.246$, and -0.418 , respectively). In addition, the expression level of the mentioned genes in the LBW infant was significantly up-regulated than the normal weight infants $(P=0.001,0.007,0.001$, and $<0.001$, respectively). (Table 3 )

Table 3. The expression level of obesity and diabetes genes in peripheral blood cells based on the birth weight 


\begin{tabular}{|llllll|}
\hline $\begin{array}{l}\text { Obesity and diabetes } \\
\text { genes }\end{array}$ & \multicolumn{2}{l}{$\begin{array}{l}\text { Spearman's rho of birth } \\
\text { weight }\end{array}$} & $\begin{array}{l}\text { Normal } \\
\text { weight }\end{array}$ & $\begin{array}{l}\text { Low birth } \\
\text { weight }\end{array}$ & P2 \\
\hline MC4R & $\mathrm{r}$ & $\mathrm{P} 1$ & & \\
\hline MTNR1B & 0.079 & 0.288 & $13.27 \pm 11.37 \star$ & $16.54 \pm 23.68$ & 0.195 \\
\hline NRXN3 & -0.221 & 0.001 & $11.72 \pm 11.90$ & $22.83 \pm 26.20$ & 0.001 \\
\hline NTRK2 & -0.011 & 0.873 & $6.93 \pm 7.46$ & $12.51 \pm 18.71$ & 0.4 \\
\hline PCSK1 & -0.235 & 0.001 & $8.02 \pm 10.52$ & $15.48 \pm 21.08$ & 0.007 \\
\hline A2BP1 & -0.246 & 0.001 & $6.23 \pm 6.39$ & $18.52 \pm 24.03$ & 0.001 \\
\hline TMEM18 & -0.096 & 0.222 & $11.32 \pm 8.34$ & $20.58 \pm 22.93$ & 0.094 \\
\hline PPAR- $\boldsymbol{N}$ & -0.027 & 0.717 & $10.19 \pm 11.12$ & $9.04 \pm 9.22$ & 0.76 \\
\hline LXR & -0.067 & 0.332 & $7.29 \pm 10.79$ & $11.90 \pm 18.09$ & 0.651 \\
\hline PTEN & 0.11 & 0.114 & $6.88 \pm 8.92$ & $4.19 \pm 5.54$ & 0.097 \\
\hline ACACB & -0.418 & $<0.001$ & $2.32 \pm 2.82$ & $49.16 \pm 48.18$ & $<0.001$ \\
\hline BDNF & -0.038 & 0.606 & $18.04 \pm 20.08$ & $22.37 \pm 26.64$ & 0.76 \\
\hline TCFL2 & -0.001 & 0.988 & $2.64 \pm 2.92$ & $2.56 \pm 2.38$ & 0.609 \\
\hline FTO & 0.081 & 0.299 & $2.40 \pm 2.74$ & $2.42 \pm 2.58$ & 0.955 \\
\hline PPAR-a & -0.06 & 0.396 & $12.81 \pm 16.14$ & $18.72 \pm 23.11$ & 0.142 \\
\hline CPT1A & 0.19 & 0.005 & $40.04 \pm 17.62$ & $21.51 \pm 22.71$ & $<0.001$ \\
\hline
\end{tabular}

*Mean \pm SD

P1 was obtained Spearman's correlation.

P2 was obtained Mann-Whitney U.

Furthermore in Table 3, we observed that the expression level of PPAR-a gene had significantly positive correlation with the birth weight $(r=0.19, \mathrm{P}=0.005)$. The expression level of PPAR-a gene in the normal weight infants was significantly up-regulated than the LBW infants $(P=0.049)$.

Liner regression showed a significant negative association between the birth weight and the expression level of obesity and diabetes genes including MTNR1B, NTRK2, PCSK1, and PTEN genes, as well as, a significant positive association between the birth weight and the expression level of PPAR-a gene. No 
significant association was seen between the birth weight and other studied expression level of obesity and diabetes genes. (Fig. 1)

\section{Discussion}

This present case- control study demonstrated that the normal birth weight had preventive role on upregulated expression level of MTNR1B, NTRK2, PCSK1, and PTEN genes, while the expression level of $P P A R$-a gene was significantly down regulated in the LBW infants compared to the infants with normal birth weight. LBW infants are at higher risk for obesity, neurodevelopment failure, cardiovascular disease, and other metabolic disorders [20]. Birth weight is considered as a suitable indicator for the quality of fetal growth and a predictor of health throughout life $[20,21]$. To best our knowledge, this current study was evaluated the association between birth weight and obesity and diabetes gene expression in healthy infants.

Our findings indicated that the birth weight was associated with up-regulated the expression level of MTNR1B. Holzapfel et al. [22] observed that the MTNR1B was associated with diabetes in children and adolescents. Another study by Lianget al. [23] showed that maternal MTNR1B genotype involved in the etiology of childhood obesity. Recently, the MTNR1B has been shown to increase the risk of obesity and type 2 diabetes $[22,24]$. It is also highly expressed in retinal cells, pancreas and especially pancreatic islet cells. Melatonin is a neurohormone secreted by pineal gland which can adjust the circadian rhythm and the circadian rhythm regulates the insulin level. However, melatonin secretion is impaired in diabetics [24].

We also indicated up-regulated the expression level of the NTRK2 with increasing of the birth weight. In study by Metrustry et al. variants in the NTRK2 gene and birth weight were examined. This study was showed that this gene was highly expressed in LBW twins [25]. NTRK2 is located on 9q21.33. NTRK2 encodes a member of the neurotrophic tyrosine receptor kinase (NTRK) family that is a membrane-bound receptor for $B D N F$ and regulates energy balance downstream of $M C 4 R$. Also it involved (involves) in MAPK pathway and cell differentiation [26]. Mutations of NTRK2 have been associated with obesity and eating behavior $[25,27]$.

In current study the expression level of the PCSK1 was up-regulated in the LBW infants. Ruiz-Narváez et al. showed that LBW plays a role the expression level of the PCSK 1 by disrupting central nervous system mechanisms and increases obesity in adulthood [28]. PCSK1 which is located on 5q15 encodes a prohormone convertase 1/3 (PC 1/3) that involved in pro-insulin processing under influencing of TCF7L2 [29]. As well as $P C S K 1$ is involved in the processing of pro-opiomelanocortin, proglucagon, proGnRH and proTRH. In addition, PCSK 1 variants are associated with extreme obesity, impaired glucose tolerance, and poly cystic ovarian syndrome. Rare mutations in PCSK 1 cause childhood obesity, impaired prohormone processing and abnormal glucose homeostasis with increasing proinsulin concentrations [28, 30].

In present study, another up-regulated the expression level of gene was related to the PTEN gene. In study by Li et al. [31] the high expression level of gene PTEN was associated with increase of insulin resistance. Although the gene was first identified as a tumor suppressor, it has recently been shown to be important 
with its antagonistic function in the insulin signaling cascade and is involved in glucose metabolism [32]. PTEN is a phosphatase which plays role in signaling pathway and suppression of tumor in which is able to suppress phosphatidylinositol 3-kinase (PI3K) signaling $[10,11,13]$. Since activation of PI3K is essential for insulin performance, PTEN is known to be effective in development of insulin resistance by reducing $\mathrm{PI} 3 \mathrm{~K}$ [33].

On the other hand, we observed that the expression level of PPAR-a gene was significantly down regulated in the LBW infants. Laleh et al. [34] demonstrated that the high expression level of PPAR-a suppressed appetite in obesity. Priego et al. [35] showed that the higher expression level of PPAR-a gene in infants is associated with a lower risk of overweight. PPAR-a is a group of legend activated nuclear receptors that are mainly expressed in tissues that are vital for fatty acid metabolism, such as the liver, kidney, and heart, where they play an important role in regulating transcription of fatty acid metabolism, lipid homeostasis, and regulation of obesity [36]. During times of starvation and energy depletion, the PPAR- $a$ increases fatty acids beta-oxidation. Animal study showed that PPAR-a deficiency was related with obesity and dyslipidemia [18].

\section{Limitations}

This current study was the first case- control study on birth weight and obesity and diabetes gene expression in healthy infants. However, it suffered from some limitations. The sample size was small due to economic constraints. Second, although we controlled many infants and maternal factors between the two groups, there was difference between the two groups because many infants in the control group were preterm in term of pregnancy weight gain and current weight infants.

\section{Conclusions}

In conclusion, this present study reflected that the expression level of MTNR1B, NTRK2, PCSK1, and PTEN genes up-regulated in the LBW infants, however, the expression level of PPAR-a gene was significantly down regulated in the LBW infants compared to the infants with normal birth weight.

\section{Abbreviations}

Low birth weight (LBW); Melanocortin 4 receptor (MC4R); Melatonin Receptor 1B (MTNR1B); Peroxisome proliferator-activated receptor gamma (PPAR- $\gamma)$; Phosphatase and tensin homolog (PTEN); Acetyl-CoA Carboxylase Beta $(A C A C B)$; peroxisome proliferator-activated receptor-a (PPAR-a), Ethylenediaminetetraacetic acid (EDTA); Neurexin-3-alpha (NRXN3); Neurotrophic Receptor Tyrosine Kinase 2 (NTRK2); Proprotein convertase 1(PCSK1); Ataxin-2 binding protein 1 (A2BP1); Transmembrane 18 gene (TMEM18); Liver $X$ receptor $(\angle X R)$; Brain-derived neurotrophic factor (BDNF); transcription factor 7-like 2 (TCF7L2); fat mass and obesity-associated (FTO); carnitine palmitoyltransferase IA (CPT1A); peripheral blood mononuclear cells (PBMC); complementary DNA (cDNA) 


\section{Declarations}

Ethical approval: All procedures performed in studies involving human participants were in accordance with the ethical standards of the institutional and/or national research committee and with the 1964 Helsinki declaration and its later amendments or comparable ethical standards. This study was approved by the Ethics Committee of Kermanshah University of Medical Sciences (ethics approval number: IR.KUMS. RES.1397.081).

Informed consent: Written informed consent was obtained from all parents after explaining the purpose of the study.

Consent for publication: not applicable.

Availability of data and materials: The datasets used and/or analyzed during the current study will be available from the corresponding author on reasonable request.

Competing interests: On behalf of all co- authors, the corresponding author states that there is no conflict of interest.

Funding: This study was supported by Kermanshah University of Medical Science (Grant No: 97155).

Author contributions: SM and SC contributed in conception and design of the research; SC and AM contributed to data collection; SM contributed to the acquisition, the analysis, and the interpretation of the data; SM and AM contributed to draft the manuscript. All authors are in agreement with the manuscript and declare that the content has not been published elsewhere

Acknowledgment: We wish to thank and acknowledge the valuable contribution of all parents in this study and especially want to acknowledge the Kermanshah University of Medical Science for financial support.

\section{References}

1. Giapros V, Vavva E, Siomou E, Kolios G, Tsabouri S, Cholevas V, et al. Low-birth-weight, but not catchup growth, correlates with insulin resistance and resistin level in SGA infants at 12 months. J Matern Fetal Neonatal Med 2017;30(15):1771-6.

2. Goldenberg RL, Culhane JF. Low birth weight in the United States. Am J Clin Nutr. 2007;85(2):584S90S.

3. Organization WH. Optimal feeding of low-birth-weight infants: technical review: World Health Organization; 2006.

4. Sharifi N, Dolatian M, Pakzad R, Yadegari L. The relationship of the structural and intermediate social determinants of health with low birth weight in Iran: A systematic review and meta-analysis. Sci J Kurdistan Uni Med Sci. 2018;23(2):21-36. 
5. Jornayvaz FR, Vollenweider P, Bochud M, Mooser V, Waeber G, Marques-Vidal P. Low birth weight leads to obesity, diabetes and increased leptin levels in adults: the CoLaus study. Cardiovasc Diabetol. 2016;15(1):1-10.

6. Zhao Y, Wang S-F, Mu M, Sheng J. Birth weight and overweight/obesity in adults: a meta-analysis. Eur J Pediatr. 2012;171(12):1737-46.

7. Khan MAB, Hashim MJ, King JK, Govender RD, Mustafa H, Al Kaabi J. Epidemiology of type 2 diabetes-global burden of disease and forecasted trends. Epidemiol Glob Health 2020;10(1):107.

8. Lim HJ, Xue H, Wang Y. Global trends in obesity. Handbook of Eating and Drinking: Interdisciplinary Perspectives. 2020:1217-35.

9. Scheen A, De Hert M. Abnormal glucose metabolism in patients treated with antipsychotics. Diabetes Metabolism. 2007;33(3):169-75.

10. Temelkova-Kurktschiev T, Stefanov T. Lifestyle and genetics in obesity and type 2 diabetes. Exp Clin Endocrinol Diabetes. 2012;120(01):1-6.

11. James D, Lessen R. Position of the American Dietetic Association: promoting and supporting breastfeeding. J Am Diet Assoc. 2009;109(11):1926-42.

12. Riancho JA, Vazquez L, Garcia-Perez MA, Sainz J, Olmos JM, Hernandez JL, et al. Association of ACACB polymorphisms with obesity and diabetes. Mol Genet Metab. 2011;104(4):670-6.

13. Beckman JA, Creager MA, Libby P. Diabetes and atherosclerosis: epidemiology, pathophysiology, and management. Jama. 2002;287(19):2570-81.

14. Abu-Elheiga L, Matzuk MM, Abo-Hashema KA, Wakil SJ. Continuous fatty acid oxidation and reduced fat storage in mice lacking acetyl-CoA carboxylase 2. Science. 2001;291(5513):2613-6.

15. Oh W, Abu-Elheiga L, Kordari P, Gu Z, Shaikenov T, Chirala SS, et al. Glucose and fat metabolism in adipose tissue of acetyl-CoA carboxylase 2 knockout mice. Proc Natl Acad Sci USA. 2005;102(5):1384-9.

16. Xu Y, Wu Y, Huang Q. Comparison of the effect between pioglitazone and metformin in treating patients with PCOS: a meta-analysis. Arch Gynecol Obstet. 2017;296(4):661-77.

17. Rangwala SM, Lazar MA. Peroxisome proliferator-activated receptor $y$ in diabetes and metabolism. Trends Pharmacol Sci. 2004;25(6):331-6.

18. Kersten S. Integrated physiology and systems biology of PPARalpha. Molecular metabolism. 2014;3(4):354-71.

19. Zarrati M, Shidfar F, Razmpoosh E, Nezhad FN, Keivani H, Hemami MR, et al. Does low birth weight predict hypertension and obesity in schoolchildren? Ann Nutrition Metabolism. 2013;63(1-2):69-76.

20. Erasun D, Alonso-Molero J, Gómez-Acebo I, Dierssen-Sotos T, Llorca J, Schneider J. Low birth weight trends in Organisation for Economic Co-operation and Development countries, 2000-2015: economic, health system and demographic conditionings. BMC Pregnanc Childbirth. 2021;21(1):1-8.

21. Jornayvaz FR, Vollenweider P, Bochud M, Mooser V, Waeber G, Marques-Vidal P. Low birth weight leads to obesity, diabetes and increased leptin levels in adults: the CoLaus study. Cardiovascular 
diabetology. 2016;15(1):73.

22. Holzapfel C, Siegrist M, Rank M, Langhof H, Grallert H, Baumert J, et al. Association of a MTNR1B gene variant with fasting glucose and HOMA-B in children and adolescents with high BMI-SDS. Euro J Endocrinol. 2011;164(2):205.

23. Liang Z, Liu H, Wang L, Chen Y, Zhou T, Heianza Y, et al. Maternal MTNR1B genotype, maternal gestational weight gain, and childhood obesity. Am J Clin Nutr. 2020;111(2):360-8.

24. Reinehr T, Scherag A, Wang HJ, Roth CL, Kleber M, Scherag S, et al. Relationship between MTNR1B (melatonin receptor 1B gene) polymorphism rs10830963 and glucose levels in overweight children and adolescents. Pediatr Diabetes 2011;12(4pt2):435-41.

25. Metrustry SJ, Edwards MH, Medland SE, Holloway JW, Montgomery GW, Martin NG, et al. Variants close to NTRK2 gene are associated with birth weight in female twins. Twin Res Hum Genet. 2014;17(4):254-61.

26. Gray J, Yeo G, Hung C, Keogh J, Clayton P, Banerjee K, et al. Functional characterization of human NTRK2 mutations identified in patients with severe early-onset obesity. Inter J Obesity. 2007;31(2):359-64.

27. Rask-Andersen M, Almén MS, Olausen HR, Olszewski PK, Eriksson J, Chavan RA, et al. Functional coupling analysis suggests link between the obesity gene FTO and the BDNF-NTRK2 signaling pathway. BMC Neuroscience. 2011;12(1):117.

28. Ruiz-Narváez EA, Haddad SA, Rosenberg L, Palmer JR. Birth weight modifies the association between central nervous system gene variation and adult body mass index. J Hum Genet 2016;61(3):193-8.

29. Pépin L, Colin E, Tessarech M, Rouleau S, Bouhours-Nouet N, Bonneau D, et al. A new case of PCSK1 pathogenic variant with congenital proprotein convertase 1/3 deficiency and literature review. J Clin Endocrinol Metab. 2019;104(4):985-93.

30. Stijnen P, Tuand K, Varga TV, Franks PW, Aertgeerts B, Creemers JW. The association of common variants in PCSK1 with obesity: a HuGE review and meta-analysis. Am J Epidemiol. 2014;180(11):1051-65.

31. Li Y-y, Xiao R, Li C-p, Huangfu J, Mao J-f. Increased Plasma Levels of FABP4 and PTEN are Associated with More Severe Insulin Resistance in Women with Gestational Diabetes Mellitus. Med Sci Mon Int Med J Exp Clin Res. 2015;21:426.

32. Yin L, Cai W-J, Chang X-Y, Li J, Zhu L-Y, Su X-H, et al. Analysis of PTEN expression and promoter methylation in Uyghur patients with mild type 2 diabetes mellitus. Medicine. 2018;97(49).

33. Maehama T, Dixon JE. The tumor suppressor, PTEN/MMAC1, dephosphorylates the lipid second messenger, phosphatidylinositol 3, 4, 5-trisphosphate. J Biol Chem. 1998;273(22):13375-8.

34. Laleh P, Yaser K, Abolfazl B, Shahriar A, Mohammad AJ, Nazila F, et al. Oleoylethanolamide increases the expression of PPAR-A and reduces appetite and body weight in obese people: A clinical trial. Appetite. 2018;128:44-9.

35. Priego T, Sanchez J, Pico C, Ahrens W, Bammann K, De Henauw S, et al. Influence of breastfeeding on blood-cell transcript-based biomarkers of health in children. Pediatric obesity. 2014;9(6):463-70. 
36. Tang Y, Vanlandingham MM, Wu Y, Beland FA, Olson GR, Fang J-L. Role of peroxisome proliferatoractivated receptor alpha (PPARa) and PPARa-mediated species differences in triclosan-induced liver toxicity. Arch Toxicol. 2018;92(11):3391-402.

\section{Figures}

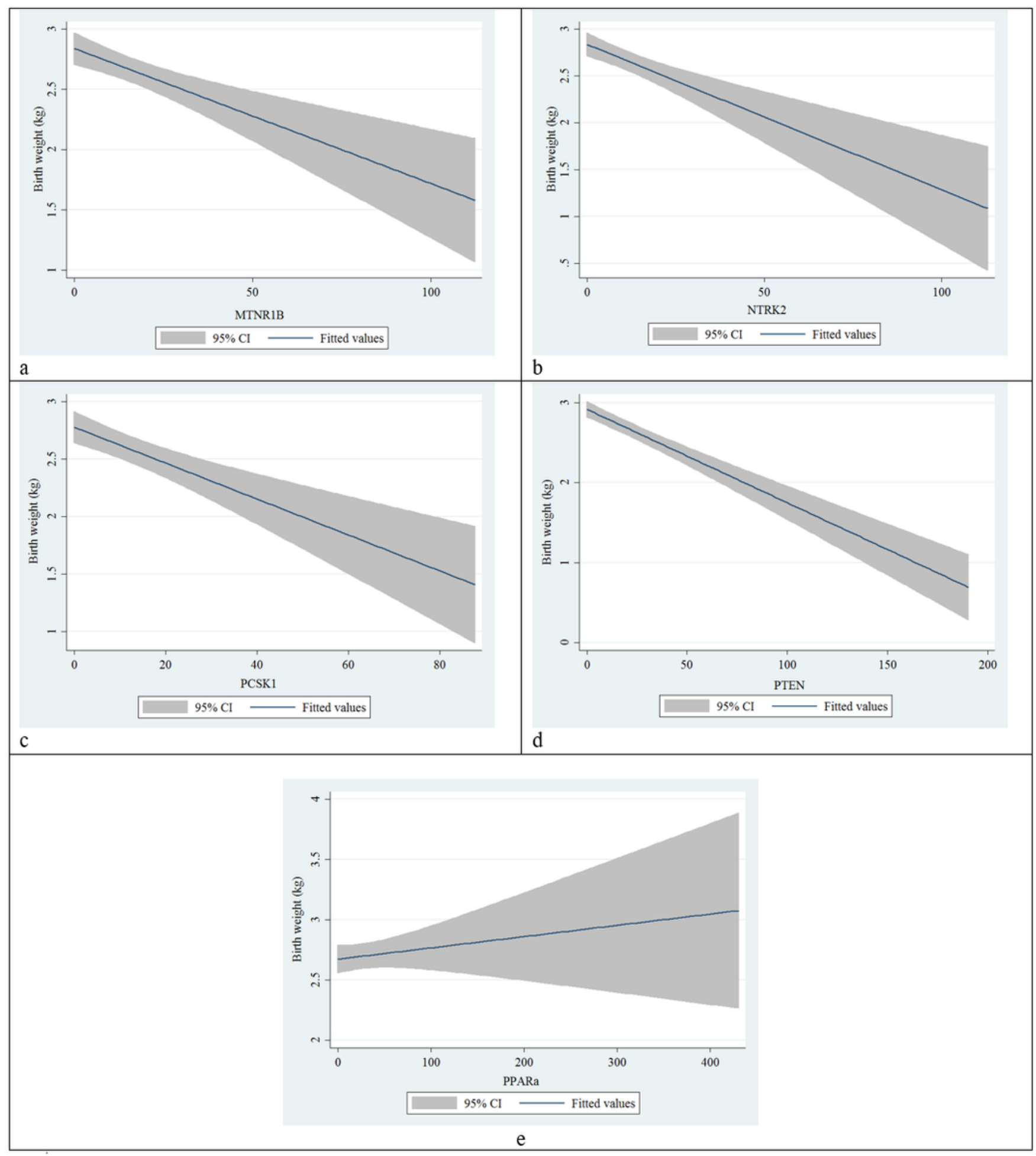


Liner regression between birth weight and obesity and diabetes genes including: a) MTNR1B, b) NTRK2, c) PCSK1, d) PTEN, d) PPAR a 